\title{
Clinical Data Interchange Standards Consortium Terminology
}

National Cancer Institute

\section{Source}

National Cancer Institute. Clinical Data Interchange Standards Consortium Terminology. NCI Thesaurus. Code C61410.

The terminology that includes terms relevant to the Clinical Data Interchange Standards Consortium. 